\title{
Research on cloud model control strategy for wind farm and energy storage system
}

\author{
Shuiming Jiang ${ }^{1, a}$, Rong Ju ${ }^{1,}$,, Kaidi Yang ${ }^{1, \text { a }}$ \\ ${ }^{1}$ School of Electrical and Automation Engineering, Nanjing Normal University, Nanjing 210042, \\ China. \\ ajsmwater@126.com
}

Keywords: wind power fluctuation, cloud model, storage of charge, low-pass filter

\begin{abstract}
This paper is based on the traditional wind-storage combined power generation system. A two-dimensional cloud model is used to control the variable weight filter to analyze the relationship between filter time constant, energy storage capacity and power fluctuation. In order to take the battery charge state into account, and reduce battery cycle times, we take both the filter power and SOC of battery storage double constraints into consideration when we stabilize the wind power fluctuations.
\end{abstract}

\section{Introduction}

With the rapid development of wind power and fan equipment technology, wind power costs tend to be reasonable. The wind farm power output is difficult to be predicted accurately because of its volatility and intermittent gained from geographical environment, weather and many other factors. Wide access to wind farms can increase uncertainly and reduce operating efficiency of the whole power system. The traditional large-capacity energy storage is generally shared by the whole network, which is important for improving the flexibility of the power grid. But it is specially designed to suppress the wind power fluctuation or improve the wind energy utilization rate in the wind-storage combined power generation system.

Some scholars have proposed a plan based on the principle of low-pass filter to stabilize the wind power control effectiveness, but it lacks energy storage system constraints. And the phase delay of low-pass filter control strategy makes the response and recovery speed slower when there is a sudden change with the battery from a extreme state. Some people have advised Kalman filter algorithm and further optimization algorithms based on it. These algorithms can effectively solve the problems caused by phase delay. However, the system process noise and measurement noise cannot be measured and the modeling is not accurate. The Kalman filter may be divergent when there is a sudden change with wind power. Other scholars have proposed an approach that views the state of charge of the battery as a constraint, and use wind power prediction method to suppress wind power fluctuations. This approach increases battery cycle times, which has adverse effects on battery life. There is a common excessive smoothing problem with all the methods above such as overcharge, over discharge and so on. Therefore, a reasonable control way to smooth wind power should take the ability of both the energy storage system to stabilize the wind power fluctuation and the energy storage system into account. It should not only prevent excessive energy storage system or over discharge, to but also extend the life of energy storage devices as far as possible.

\section{D CMC: Two- Dimensional cloud model controller based on the variable weight average filter}

A 2D CMC is designed to control the variable weight average filter based on Matlab/ Simulink to suppress wind power fluctuation.

Storage system. As is shown in Figure 1, we put a storage system at the bus exit of the wind farm to suppress wind power fluctuations. In comparison with traditional low-pass filters, the variable weight average filter shows a great advantage. 


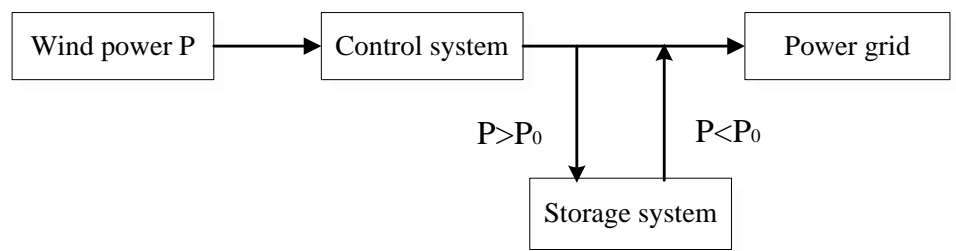

Fig. 1 The structure of wind farm storage system

We hope that the wind farm connects to the power grid at a stable power P. And we know wind farm real-time power is $P_{0}$.

If $P_{0}>\mathrm{P}$, store excess power to the energy storage system; if $P_{0}<\mathrm{P}$, the energy storage system provide more power to make it sufficient and approximate.

Double - constrained cloud model control for wind - storage combined system. As is shown in Figure 2, the battery storage system based on the variable weight average filter help adjust wind power before it connects to power grid. A 2D CMC is designed to help variable weight average filter control the system adaptively. We have 2 constraints which is volatility and SOC (battery state of charge) of the 2D CMC.

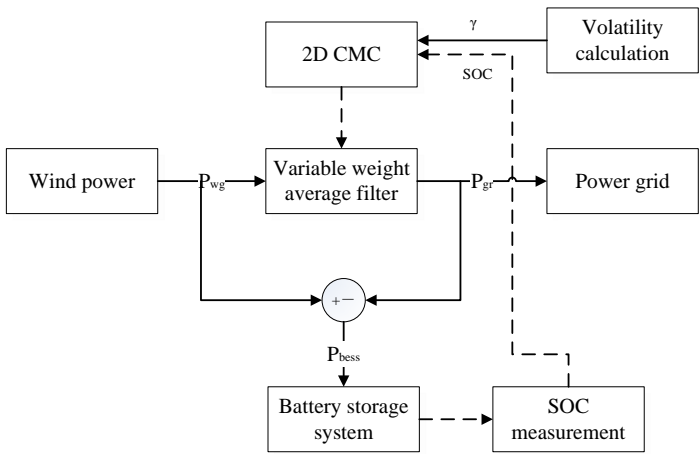

Fig. 2 The structure of 2D CMC for wind - storage combined system

The cloud model controller. The structure of multi-rule cloud model controller is shown in Figure 3. We use two-dimensional cloud model controller in this paper, which means we should make $n=2$.

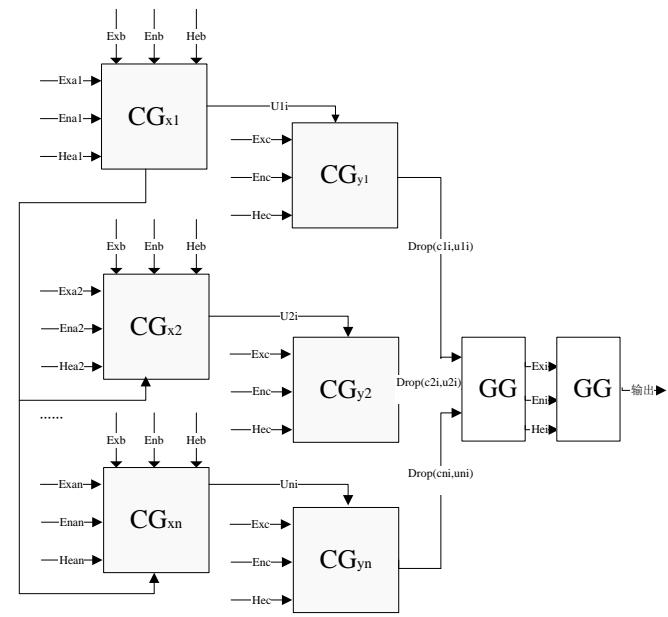

Fig. 3 The structure of multi-rules cloud controller

Here are the steps for designing:

(1) Define and normalize the former and consequent for cloud model. The input of the controller is $\gamma$ and SOC, as the former. The output is $\mathrm{N}$, as the consequent. The value of $\gamma$ ranges from -1 to 1 , SOC from 0 to $1, \mathrm{~N}$ from 20 to 50 considering the relationship between volatility and energy storage capacity, normalization ranging from 0 to 1.

(2) Build cloud models for these variables. the expectations (Ex) of $\gamma, \mathrm{SOC}$ and N are divided into corresponding parts according to the bisection principle. In accordance with the "3En Principles", the value of the 3 entropies (En) are the 1/3 of their expectations. And we adjust the value of their high entropies (He) according to the control effect. The detailed values of volatility $(\gamma)$, SOC, and filter coefficient are show in Table 1. And their cloud models are shown in Figure. 4. 


\begin{tabular}{|c|c|c|}
\hline & Extremely minus & $\mathrm{A} 1(-1,0.5 / 3,0.15)$ \\
\hline & Slightly minus & $\mathrm{A} 2(-0.5,0.5 / 3,0.15)$ \\
\hline \multicolumn{3}{|l|}{ Volatility } \\
\hline & Zero & $\mathrm{A} 3(0,0.5 / 3,0.15)$ \\
\hline \multicolumn{3}{|l|}{$(Y)$} \\
\hline & Slightly plus & $\mathrm{A} 4(0.5,0.5 / 3,0.15)$ \\
\hline
\end{tabular}

Former

Extremely plus

Low

SOC

Moderate

High
$\mathrm{A} 5(1,0.5 / 3,0.15)$

$\mathrm{B} 1(0,0.5 / 3,0.02)$

$\mathrm{B} 2(0.5,0.5 / 3,0.02)$

$\mathrm{B} 3(1,0.5 / 3,0.02)$

$\mathrm{C} 1(0,0.25 / 3,0.01)$

Extremely low

$\mathrm{C} 2(0.25,0.25 / 3,0.01)$

Consequent

Filter coefficient $\mathrm{N}$

Moderate

C3(0.5, 0.25/3, 0.01)

Slightly high

$\mathrm{C} 4(0.75,0.25 / 3,0.01)$

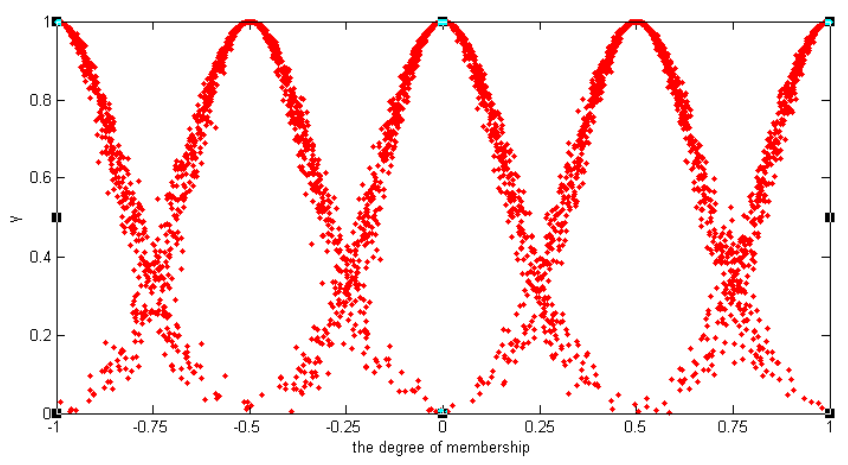

a. Cloud model for $\gamma$

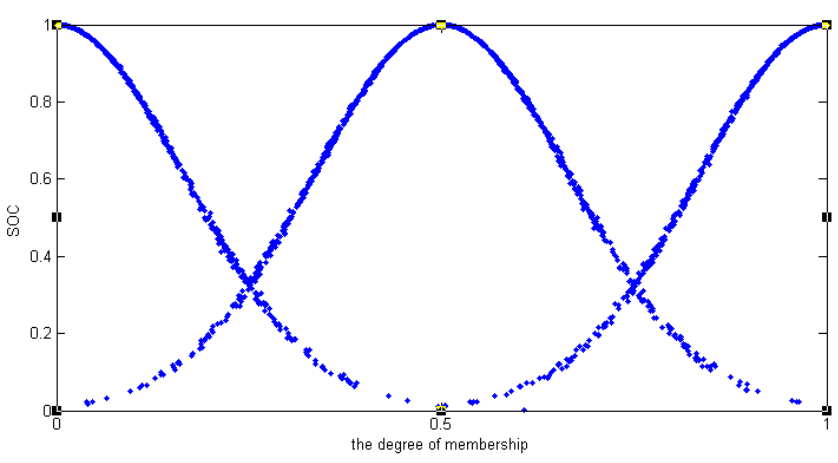


b. Cloud model for SOC

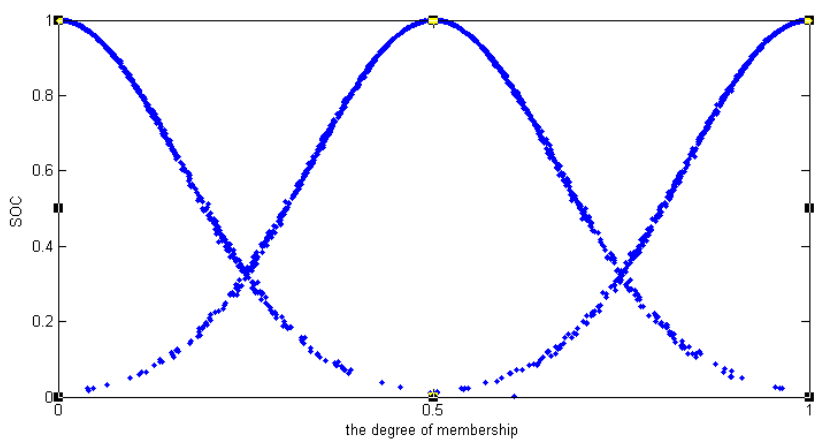

c. Cloud model for $\mathrm{N}$

Figure. 4 Cloud models for former and consequent

(3) Build up a library for logic rules. The library is based on the characteristics of wind power output and energy storage system. If $\gamma$ is extremely plus or extremely minus, we increase $\mathrm{N}$; if $\gamma$ is slightly plus, slightly minus or zero, we reduce $\mathrm{N}$; if SOC level is moderate, then $\mathrm{N}$ value is also moderate. We designed 15 two-dimensional cloud model inference rules in total, shown by matrix (1).

$$
R=\left\{r_{i j}\right\}=\left[\begin{array}{lll}
2 & 4 & 5 \\
1 & 3 & 3 \\
1 & 1 & 1 \\
3 & 3 & 1 \\
5 & 4 & 2
\end{array}\right]
$$

$r_{i j}$ means the rule: if $\mathrm{A}=A_{i}, \mathrm{~B}=B_{j}$, then $\mathrm{C}=C_{k} ; \mathrm{i}=1 \sim 5, \mathrm{j}=1 \sim 3, \mathrm{k}=r_{i j}$.

For example, $r_{21}$ means : if $\mathrm{A} 2$ and $\mathrm{B} 1$ then $\mathrm{C} 1$; if $\gamma$ is extremely minus, and $\mathrm{SOC}$ is low, then $\mathrm{N}$ should be low.

The two - dimensional cloud model and the degree of membership are shown in Figure. 5.

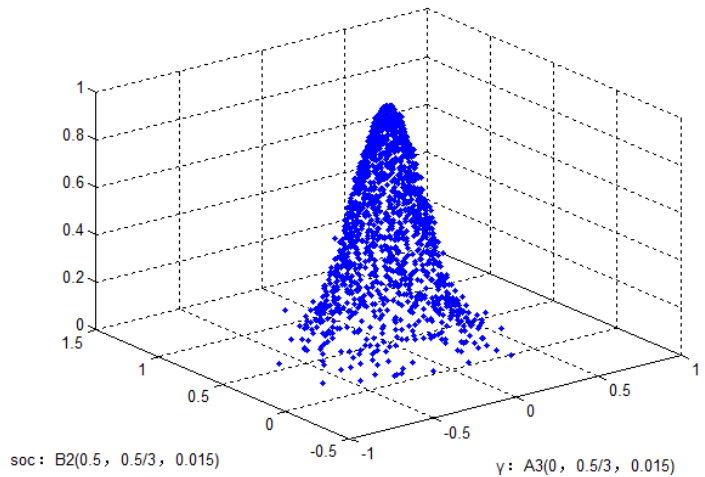

Fig. 5 Two - dimensional cloud model and the degree of membership

\section{Conclusion}

Compared to waveform smoothed by the first-order low-pass filter and the waveform without being smoothed, the waveform smoothed by the two - dimensional cloud model shows a great advantage. As can be seen from Figure 6, this method can improve the low-pass filter phase delay problem to a large extent, and can also take stabilization into account. 


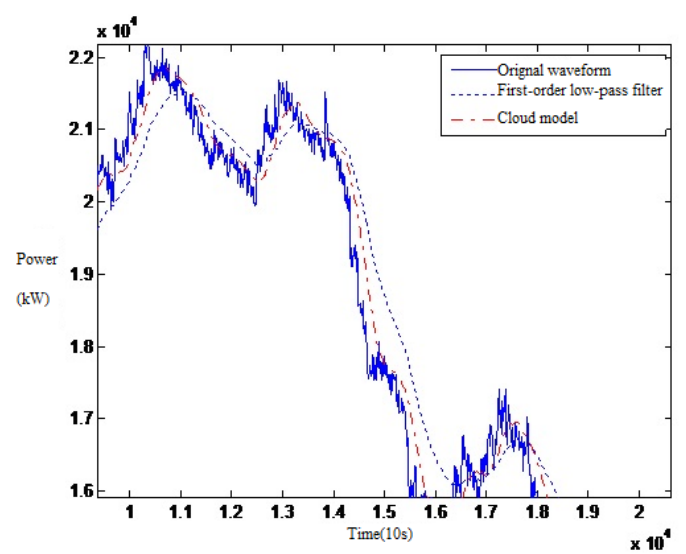

Fig. 6 Comparison and results among different smoothing methods

\section{References}

[1] Zhang Xinsong, Gu Juping, Yuan Yue, et al. Strategy of Smoothing Wind Power Fluctuation Based on Battery Energy Storage System[J] Proceedings of the CSEE, 2014, 34(28): 4752- 4760.

[2] Zhou Qicong, Xu Yaoliang, Chu Shichong, et al. A New Strategy for Smoothing Wind Power Fluctuation Based on Battery Energy Storage System[J] Modern Electric Power, 2016,33(2): 59- 63.

[3] Sun Chengchen, Yuan yue, Choi San Shing, et al. Capacity Optimization and Operational Strategy of the Wind-Energy Storage Hybrid Systems for Energy Dispatching[J] Power System Technology, 2015,39(8): $2107-2114$.

[4] Wang Gang, Qiu Xiaoyan, Zhang xiaochen, et al. Optimized Control Strategy for Battery Energy Storage System to Smooth Wind Power Output[J] Proceedings of the CSU-EPSA,2015,27(8):66- 70.

[5] Li Peiqiang, Li Wenying, Tang Jie, et al. Method of Hybrid Energy Storage to Smooth the Fluctuations for Wind Power Based on State of Charge Optimization[J] Proceedings of the CSU-EPSA, 2017,29(3): 20- 27.

[6] Lei Ting, Ouyang ZengkAi, Li Zheng, et al Coordinated control of battery SOC maintaining and filtering for wind power fluctuation smoothing[J] Electric Power Automation Equipment,2015,35(7): 126-131. 